\title{
A importância do farmacêutico na consulta à pacientes pediátricos em uso de
}

\section{medicamentos off-Label}

The importance of the pharmacist in consulting pediatric patients using off-label medications

La importancia del farmacéutico en la consulta de pacientes pediátricos que utilizan medicamentos fuera de etiqueta

\author{
Daiane Pereira dos Santos Menese \\ ORCID: https://orcid.org/0000-0001-7618-6401 \\ Faculdade Integrada Carajás, Brasil \\ E-mail: redededfarma@gmail.com \\ Carolinne de Oliveira Marquez \\ ORCID: https://orcid.org/0000-0001-6556-5094 \\ Faculdade Integrada Carajás, Brasil \\ E-mail: carolzinhaoliveiramarquez@yahoo.com.br
}

\begin{abstract}
Resumo
Os medicamentos tem papel fundamental na recuperação e manutenção da saúde, devendo ser seguros, eficazes, de qualidade e acessíveis para o paciente. A consulta farmacêutica aos pacientes pediátricos em uso de medicamentos off-Label cuja utilização se dá de forma diferente da descrita em seu rótulo ou bula, quando se fala em indicação, via de administração, posologia e faixa etária, tem o papel de auxiliar na farmacoterapia, minimizando os erros e as reações adversas ao medicamento indicados por médicos e prevenir problemas relacionados aos medicamentos (PRM's). A administração de medicamentos de adultos em crianças é uma prática comum que deve ser tratada com cautela, pois a fisiologia da criança é distinta e os riscos de intoxicação são diversos. O objetivo desse estudo foi evidenciar a importância do farmacêutico no acompanhamento farmacoterapêutico à pacientes pediátricos em uso de medicamentos off-Label durante as consultas farmacêuticas. A busca ocorreu no banco de dados da Literatura LatinoAmericana e do Caribe em Ciências da Saúde (LILACS) e no Scientific Eletronic Library Online (SciELO). Para delimitação dos conteúdos foram utilizados critérios de inclusão: artigos disponíveis na íntegra em português e inglês, no período de 2012 a 2021 com acesso gratuito e que tivessem pertinência com tema. Os resultados demonstraram que o farmacêutico é imprescindível no acompanhamento e na orientação dos pacientes que usam medicamentos offLabel, sendo necessária a adequada formação desse profissional sobre a temática. Nesse contexto, o farmacêutico surge como profissional que promove o uso correto dos fármacos em pediatria, contribuindo para uma farmacoterapia segura.
\end{abstract}

Palavras-chave: Consulta; Farmacêutico; Pediatria; Off-Label.

\begin{abstract}
Medicines play a fundamental role in the recovery and maintenance of health, and must be safe, effective, quality and accessible to the patient. The pharmaceutical consultation for pediatric patients using off-label drugs whose use is different from that described on their label or package insert, when it comes to indication, route of administration, dosage and age group, has the role of assisting in pharmacotherapy, minimizing errors and adverse drug reactions indicated by physicians and preventing drug-related problems (PRM's). The administration of medications from adults to children is a common practice that should be treated with caution, as the child's physiology is different and the risks of intoxication are diverse. The aim of this study was to highlight the importance of the pharmacist in the pharmacotherapeutic monitoring of pediatric patients using off-label medications during pharmaceutical consultations. The search took place in the database of the Latin American and Caribbean Literature in Health Sciences (LILACS) and in the Scientific Electronic Library Online (SciELO). To delimit the contents, inclusion criteria were used: articles available in full in Portuguese and English, in the period from 2012 to 2021 with free access and that were relevant to the theme. The results showed that the pharmacist is essential in the monitoring and guidance of patients who use offlabel drugs, requiring the proper training of this professional on the subject. In this context, the pharmacist appears as a professional who promotes the correct use of drugs in pediatrics, contributing to safe pharmacotherapy.
\end{abstract}

Keywords: Query; Pharmacist; Pediatrics; Off-label. 


\begin{abstract}
Resumen
Los medicamentos juegan un papel fundamental en la recuperación y mantenimiento de la salud, y deben ser seguros, efectivos, de calidad y accesibles para el paciente. La consulta farmacéutica para pacientes pediátricos que utilicen medicamentos off-label cuyo uso sea diferente al descrito en su etiqueta o prospecto, en cuanto a indicación, vía de administración, posología y grupo de edad, tiene la función de auxiliar en la farmacoterapia, minimizando errores. y reacciones adversas a medicamentos indicadas por médicos y prevención de problemas relacionados con medicamentos (PRM). La administración de medicamentos de adultos a niños es una práctica común que debe tratarse con precaución, ya que la fisiología del niño es diferente y los riesgos de intoxicación son diversos. El objetivo de este estudio fue resaltar la importancia del farmacéutico en el seguimiento farmacoterapéutico de los pacientes pediátricos que utilizan medicamentos off-label durante las consultas farmacéuticas. La búsqueda se realizó en la base de datos de la Literatura Latinoamericana y del Caribe en Ciencias de la Salud (LILACS) y en la Biblioteca Científica Electrónica en Línea (SciELO). Para delimitar los contenidos se utilizaron criterios de inclusión: artículos disponibles íntegramente en portugués e inglés, en el período de 2012 a 2021 con acceso gratuito y que fueran relevantes para la temática. Los resultados mostraron que el farmacéutico es fundamental en el seguimiento y orientación de los pacientes que utilizan fármacos off-label, requiriendo la adecuada formación de estos profesionales en el tema. En este contexto, el farmacéutico aparece como un profesional que promueve el uso correcto de los medicamentos en pediatría, contribuyendo a una farmacoterapia segura.
\end{abstract}

Palabras clave: Consulta; Farmacéutico; Pediatría; Fuera de etiqueta.

\title{
1. Introdução
}

O termo off-Label inclui diversas situações da prática diária em saúde, para Porto (2015) engloba administração de doses elaboradas a partir de especialidades farmacêuticas registradas, uso de medicamentos importados e substâncias químicas sem grau farmacêutico, indicações e posologias não usuais; administração do medicamento por vias diferentes das preconizadas, administração em faixas etárias para as quais o medicamento não foi testado; administração para o tratamento de doenças que não foram estudadas e indicações terapêuticas diferentes das aprovadas para o medicamento.

Os medicamentos exercem um papel fundamental na recuperação e manutenção da saúde, devendo ser seguros, eficazes, de qualidade e ao menor custo possível para o paciente e para a comunidade. Eles garantem qualidade de vida para o usuário (Maniero, 2018).

De acordo com Carvalho (2016) uma prescrição off-Label é caracterizada quando um medicamento é usado fora de suas indicações terapêuticas, não respeitando posologia e dosagem. Isso acontece quando não há aprovação de um fármaco para certa população ou para doença crônica terminal, sendo uso muito comum na pediatria, obstetrícia, geriatria ou a psiquiatria, devido à escassez de ensaios clínicas para tais classes.

Ao serem prescritos pelos médicos, aos pacientes sendo os mesmos adultos, idosos, gestantes ou crianças devem-se considerar as características como qualidade, o custo-efetividade e a segurança. Assim, estudos do tipo ensaios clínicos são necessários para uma prescrição segura, principalmente nos grupos de pacientes grávidas, idosos e crianças (Soares, 2020).

Para Diel (2020) o uso off-Label de medicamentos caracteriza-se pelo uso do produto em situação diferente da orientada pela agência reguladora do país com respeito à idade, à dose, à indicação ou à via de administração, a grande parte dos medicamentos tem em sua indicação o uso pediátrico acima de 2 anos ou ainda uso adulto e pediátrico não sendo orientado a idade mínima para administração em crianças.

O uso racional de medicamentos (URM) é indicado para toda faixa etária, mas é no uso pediátrico que se deve dobrar a atenção e a promoção à saúde e ao uso consciente dos fármacos, porém as principais causas de internações ou de consultas são por PRM's - Problemas Relacionados aos Medicamentos, sendo causados por automedicação (Marques, 2017).

Estudos evidenciam que o farmacêutico clínico presente em equipes multiprofissionais de saúde garante a segurança e reduz erros relacionados ao uso de medicamentos, sendo capaz de avaliar as prescrições, dispensações e administrações, além de acompanhar as reações adversas (Lucena, 2018). 
O papel do farmacêutico sendo o profissional mais acessível é o de tornar o uso do medicamento mais seguro podendo realizar o acompanhamento farmacoterapêutico individualizado de acordo com a necessidade de cada paciente (Nascimento, 2020).

Conforme Vieira (2021) a atenção farmacêutica é muito importante e o farmacêutico é o profissional capacitado para de perto acompanhar e registrar as reações adversas relacionadas ao uso off-Label dessas medicações que por não terem comprovações científicas elevam os riscos de efeitos indesejáveis. Durante a consulta farmacêutica o cuidador em um consultório terá esclarecida todas as questões sobre a saúde da criança e através de medidas não - farmacológicas melhorará o quadro clínico do paciente.

O objetivo desse artigo é evidenciar a importância do farmacêutico na consulta à pacientes pediátricos com uso de medicamentos off-Label, público esse que é bastante vulnerável a doenças e o uso desse medicamento é indispensável a boa condução de seu tratamento para que as crianças tenham uma excelente qualidade de vida.

\section{Farmacologia pediátrica}

Paiva (2017) descreve que a criança ao nascer tem que se adaptar ao mundo e a tudo o que ele lhe oferece, muitas vezes algumas coisas fogem ao "normal" do seu organismo e ele reage com o surgimento de doenças e as mesmas devem ser tratadas. Para as crianças algumas medicações tem sua segurança confirmada, mas algumas classes de medicamentos ainda são utilizadas em uso off-Label, pois a alternativa mais viável para a melhora da condição clínica é essa administração.

Com o amadurecimento do corpo a criança tem as mudanças na idade, peso, altura e as modificações em relação à farmacocinética e farmacodinâmica vão surgindo e com todas essas alterações a escolha da via e da forma farmacêutica ideal para cada faixa etária, pois essas contarão muito no momento da metabolização dos medicamentos (Medeiros, 2018).

O termo criança pode ser entendido de modo genérico, mas as idades são divididas e podem ser compreendidas no Quadro 1, na classificação da população pediátrica adotada pela FDA e EMA.

Quadro 1: Classificação da população pediátrica adotada pela FDA e EMA.

\begin{tabular}{|l|l|l|}
\hline & FDA & EMA \\
\hline Intrauterino & Da concepção até ao nascimento & \\
\hline Recém - nascido & Do nascimento até 1 mês & Termo: 0 a 27 dias \\
\hline Lactente & 1 mês a 2 anos & $\begin{array}{l}\text { Lactente e criança pequena } 28 \text { dias a } \\
23 \text { meses }\end{array}$ \\
\hline Criança & 2 anos ao início da puberdade & 2 anos a 11 anos \\
\hline
\end{tabular}

FDA = Food and Drug Administration $\quad$ EMA = European Medicines Agency. Fonte: Adaptado de Medeiros, (2018). 


\section{Medicamentos de uso off-label}

Os medicamentos de uso off-Label são fármacos registrados, mas que não são indicados em sua bula oficial a posologia para faixa etária especificada durante seu registro no órgão, podem ser utilizados em doenças não estudadas ou quando não se encontram outras alternativas para o tratamento (Medeiros, 2020).

Profissionais da saúde trabalham juntos no desenvolvimento e aplicação de métodos clínicos e epidemiológicos para avaliar os benefícios e riscos potenciais de qualquer tratamento, seja farmacológico ou não farmacológico que dispõem de meios considerados excepcionais na recuperação do paciente (Silva, 2021).

Quando um medicamento não está regulamentado ou introduzido no mercado para uso em determinada faixa etária, não obrigatoriamente este não pode ser utilizado, apenas não foram realizados testes específicos para seu uso (Afonso, 2015).

O uso off-Label e não licenciado é uma prática generalizada, crescente e internacional que preocupa profissionais de saúde e pacientes por causa da falta de segurança, expondo crianças a riscos potencialmente desnecessários, considerando que o profissional assume a responsabilidade por esse ato (Diel, 2020).

Com a falta de testes específicos é insuficiente o embasamento científico sobre a farmacocinética e a farmacodinâmica de medicamentos nesse grupo de indivíduos, surgindo reações adversas graves e imprevisíveis (Kozma, 2021).

\section{O uso inadequado do medicamento off-Label em crianças}

O uso inadequado de medicamentos em crianças tem sido evidenciado em diversos estudos, principalmente quando é levado em consideração a automedicação e as intoxicações medicamentosas (Maniero, 2018).

O uso de medicamentos off-Label envolve muitos fatores, o uso adulto é adaptado para serem administrados em crianças, quando se utiliza de determinada forma farmacêutica e realiza adaptações para que possa ser administrada em crianças. Para Loureiro (2015) chama a atenção vários problemas que podem ocorrer e envolvem a estabilidade físico-química, microbiológica e a própria biodisponibilidade, podendo acarretar efeitos adversos e intoxicações.

Os pacientes pediátricos são classificados como órfãos terapêuticos, ou seja, por motivos legais, éticos e econômicos as crianças não são incluídas na maioria dos ensaios clínicos, sendo o uso de medicamentos feito de modo empírico ou mesmo off-Label (Diel, 2020).

O uso desses medicamentos em crianças é muito perigoso e considerado uma prática muito arriscada, por não haver estudos e testes realizados que garantam sua efetividade e sucesso na terapêutica, podendo o mesmo causar intoxicações (Medeiros, 2020).

A falta de estudos e ensaios clínicos voltados para uso pediátrico, que garantiria uma resposta confiável com uso adequado e eficácia e segurança garantidas quanto a utilização do fármaco na pediatria, eleva a utilização de formas farmacêuticas testadas e aprovadas para uso adulto em crianças das variadas faixas etárias (Loureiro, 2015).

Muitos estudos evidenciam o alto índice de reações adversas a medicamentos (RAM) no público infantil; essa incidência ocorre devido ao uso de medicamentos sem estudos comprovados que assegure seu uso pediátrico (Carvalho, 2016.

\section{Cálculos de dose}

Da Gama (2021) analisa cada indivíduo em sua existência, onde apresentam diferenças fisiológicas e a resposta terapêutica aplicada consequentemente muda, tornando-a individualizada. O uso de drogas exclusivas para adultos coloca a população de neonatos e pediátricas vulneráveis, aumentando os riscos para esses pacientes, daí a importância da formulação e estudos para produção de novos medicamentos de uso pediátrico. 
De acordo com o estudo de Loureiro (2015), os profissionais médicos devem assegurar em suas prescrições que a terapia escolhida seja segura e eficaz. A dose para crianças não deve levar em consideração apenas a relação peso/altura. Pois outros fatores fisiológicos estão envolvidos e alguns métodos e cálculos podem ser utilizados, no ato da prescrição o médico deverá também orientar os cuidadores sobre o uso dessa medicação que de acordo com a idade da criança seria contra indicado, esse esclarecimento deve ser realizado também durante a consulta farmacêutica.

Estudos evidenciam que o método mais seguro para adaptar o uso de formulações medicamentosas de uso adulto em crianças é a que utiliza como regra a área da superfície corporal como base para obter a dose ideal, dentro dos padrões fisiológicos de cada criança (Marques, 2017).

Desse modo, os medicamentos utilizados na pediatria podem ser indicados para finalidades distintas das recomendadas. Medeiros (2018) emprega o termo off-Label no uso dos fármacos em faixa etária, patologia, modo e frequência da administração diferentes das analisadas durante os ensaios clínicos. Outras empregabilidades dos medicamentos aplicadas à faixa etária infantil é a modificação de formas farmacêuticas quando há ausência da forma comercial do fármaco requerido ou a importação de medicamentos de outros países.

Seria ideal se todos os medicamentos disponíveis para uso adulto estivessem também disponíveis para utilização em todas faixas etárias, mas por falta de estudos são necessárias adaptações das doses, tendo como base a posologia para adultos, por meio de fórmulas, como demonstrado na Figura 1, que apresenta a regra de Young, regra de Law e regra de Clark (Medeiros, 2020).

Quadro 2 - Cálculo de ajustes de doses na pediatria.

\begin{tabular}{|c|c|c|}
\hline Nome da fórmula & Idade ou peso do paciente & Fórmula \\
\hline Regra de Clark & $<30 \mathrm{~kg}$ & $\mathrm{DP}=\underline{\text { Peso }(\mathrm{kg}) \times \mathrm{DA}}$ \\
\hline Regra de Law & $<1$ ano & $\mathrm{DP}=\underline{\mathrm{Idade}(\mathrm{meses}) \times \mathrm{DA}}$ \\
\hline Fórmula de Young & De 1 a 12 anos & $\mathrm{DP}=\underline{\text { Idade (anos) } \mathrm{DA}}$ \\
\hline
\end{tabular}

DP $=$ Dose pediátrica; DA = Dose do adulto. Fonte: Medeiros, (2020).

Essas regras são comumente aplicadas e muitas vezes necessitam de adaptações. As crianças atuais estão cada vez mais sensíveis e iniciando a utilização precoce de medicamentos, cuja prescrição não é discriminada em bula para sua idade, devendo ter uma base na literatura para dar subsídio ao clínico a melhor posologia a ser administrada (Marques, 2017).

\section{Consulta clínica farmacêutica}

A consulta clínica farmacêutica é complexa e tem como objetivo auxiliar a farmacoterapia, além de promover o uso racional de medicamentos onde o farmacêutico presta atendimento direto ao paciente, nesse caso pediátrico e ao cuidador, acompanha sua evolução garantido a adesão aos tratamentos, visando a promoção, proteção e recuperação da saúde (Rocha et al., 2020).

O farmacêutico deve preparar os pacientes para administrar corretamente o fármaco, e acompanhá-los para que o processo de adaptação seja facilitado. Trabalhar com medicações com crianças exige expertise para que se torne um momento 
tranquilo, com a colaboração da criança que deverá confiar no profissional, evitando assim, dúvidas, dificuldades e possíveis complicações no uso correto do medicamento (Silva Júnior, 2019).

Em geral os pais ou responsáveis levam suas crianças às farmácias antes mesmo de irem ao hospital por ser um meio mais rápido de acesso ao cuidado, tendo como o profissional a sua disposição o farmacêutico que fará a anamnese, poderá realizar a triagem e, quando necessário, encaminhará o paciente para um profissional adequado à sua situação (Vieira, 2021).

\section{Atenção farmacêutica ao paciente pediátrico}

Para Reis et al. (2018) os serviços prestados pelo farmacêutico clínico são direcionados aos pacientes de forma individualizada, rastreando doenças pré-existentes, realizando a revisão da farmacoterapia se necessário ou o manejo de problemas autolimitados, realizando a educação em saúde de forma plena, o que em muitos casos a falta desses conhecimentos levam ao agravamento dos problemas a serem tratados.

Ele é o responsável por promover o Uso Racional de Medicamentos (URM), prevenir a automedicação, além de detectar os problemas relacionados a medicamentos (PRM's), auxiliando na revisão da farmacoterapia. Muitas são as prescrições que possuem polifarmácia, o que dificulta a conciliação de toda a medicação, que deverá ter o conhecimento do farmacêutico (Nascimento, 2020).

O farmacêutico dentro das suas habilidades deverá analisar criteriosamente a dose e regime posológico adequados, na escolha da forma farmacêutica mais indicada para a criança, das possíveis técnicas de administração, e nos conhecimentos relativos à farmacovigilância e ensaios clínicos (Medeiros, 2018).

Considerando a alta prevalência do uso de medicamentos em crianças, assim como a prática da automedicação por seus cuidadores, Mariero (2018) reconhece a importância da promoção do uso racional de fármacos para essa população que em vários casos possuem grande quantidade de medicamentos em seus lares e utilizam sem ter a certeza de sua real necessidade.

Silva Júnior (2019) descreve outro problema sério na pediatria, garantir a adesão e a administração dos medicamentos pelos cuidadores, que em grande parte são as mães ou avós que tem dificuldades, seja por falta de escolaridade, dificuldade na escrita médica e/ou na garantia do acesso ao medicamento para dar início ao tratamento proposto. Lembrando que o farmacêutico acaba sendo o profissional primordial na adesão deste tratamento.

Paiva (2017) destaca situações observadas diariamente nos consultórios onde crianças chegam com febre e os pais administram medicamentos de uso adulto por só terem estes disponíveis em sua residência, como a nimesulida, que em sua embalagem indica o uso pediátrico, porém acima de 12 anos, o que a torna inviável para crianças abaixo dessa faixa etária. Diversos medicamentos pediátricos são questionáveis como no caso dos antitérmicos, por exemplo, que dispõem de poucos estudos a respeito de sua eficácia e segurança, onde apenas dipirona, ibuprofeno e diclofenaco são indicados para a pediatria.

Lubrano (2016) discute que o paracetamol assim como os demais medicamentos destacados anteriormente também é um antitérmico seguro podendo ser administrado em crianças menores de 2 anos.

\section{Metodologia}

O presente estudo trata-se de uma revisão integrativa. Considera-se uma revisão integrativa um método que permite a síntese de conhecimento e a incorporação dos resultados de estudos significativos na prática (de Sousa, 2017), sendo fundamentada na pesquisa bibliográfica sobre a importância do farmacêutico na consulta à pacientes pediátricos com uso de medicamentos off-Label. Para consolidação da mesma seguiu-se as seguintes etapas: escolha do tema, levantamento 
bibliográfico preliminar, formulação do problema, busca de fontes, leitura do material, organização lógica do assunto e, por fim, redação do texto.

A coleta foi realizada no período de janeiro a setembro de 2021, onde foram investigados estudos publicados nas bases de dados: Literatura Latino-Americana e do Caribe em Ciências da Saúde (LILACS) e no Scientific Eletronic Library Online (SciELO), empregando-se os descritores "consulta", "farmacêutico", "pediatria" e "off-Label", referente ao período de 2015 a 2021.

Foram incluídos artigos completos em português e inglês, de 2015 e 2021, que tratavam da temática em questão e que estavam disponibilizados eletronicamente na íntegra. Foram excluídos os artigos publicados anteriormente a 2015 e que não estivessem condizentes com o tema abordado.

Ao iniciar a pesquisa foram encontrados 50 artigos relacionados a importância do farmacêutico na consulta à pacientes pediátricos com uso de medicamentos off-Label, dos quais 30 eram duplicatas, ficando 20 artigos para a leitura. Durante o processo de análise, 10 artigos foram eliminados por não corresponderem as principais adaptações dos critérios estabelecidos, resultando em 10 artigos selecionados de acordo com a abordagem e objetividade do tema na elaboração do artigo. O fluxograma da Figura 1 demostra de forma transparente todas as metodologias de busca pelas pesquisas escolhidas para a confecção da respectiva revisão.

Figura 1: Seleção de artigos

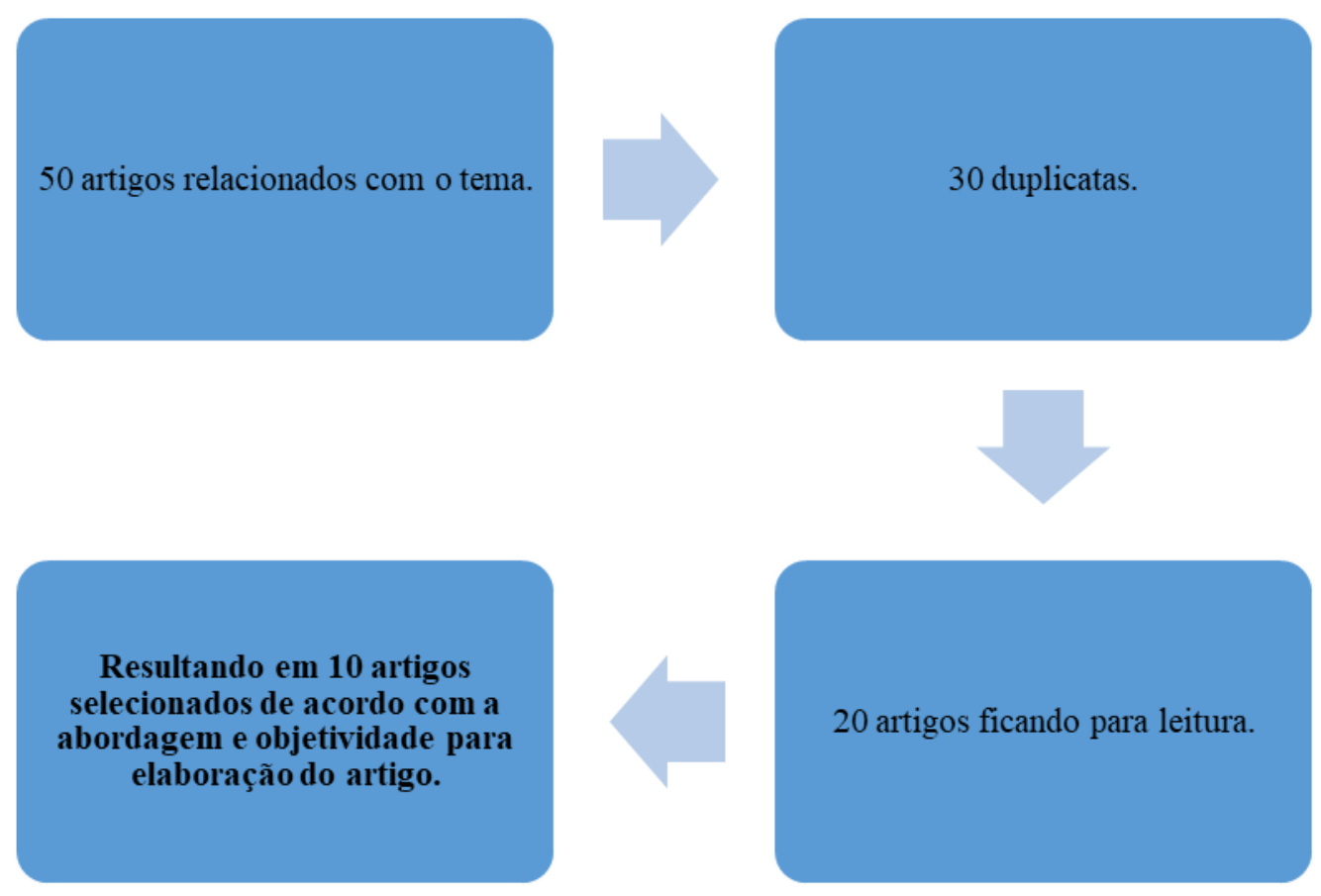

Fonte: Autoras, 2021.

\section{Resultados}

Após a análise dos artigos foram selecionados 10 estudos para integrar este artigo de revisão. A Tabela 1 apresenta os textos selecionados e sua distribuição por autor, ano, objetivo, desenho de estudo e considerações finais. 
Research, Society and Development, v. 10, n. 15, e208101522896, 2021

(CC BY 4.0) | ISSN 2525-3409 | DOI: http://dx.doi.org/10.33448/rsd-v10i15.22896

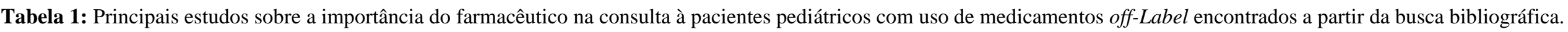

\begin{tabular}{|c|c|c|c|}
\hline AUTOR/ANO & OBJETIVO & DESENHO DO ESTUDO & CONSIDERAÇÕES FINAIS \\
\hline Afonso (2015). & $\begin{array}{l}\text { Contribuir para um melhor conhecimento das } \\
\text { variáveis associadas à utilização de } \\
\text { medicamentos em Pediatria. }\end{array}$ & Revisão Bibliográfica. & $\begin{array}{l}\text { O farmacêutico clínico, pelos seus conhecimentos sobre medicamentos e o seu } \\
\text { papel no processo de distribuição de fármacos, encontra-se numa posição } \\
\text { fundamental para a prevenção dos erros de medicação e contribuir para o uso } \\
\text { correto da medicação. Entre as principais intervenções do farmacêutico clínico } \\
\text { incluem-se a prestação de informação acerca do medicamento aos restantes } \\
\text { profissionais de saúde, quer sejam questões acerca do manuseamento ou de } \\
\text { interações, sugestões acerca do tratamento e prevenção de erros de medicação. }\end{array}$ \\
\hline Loureiro (2015). & $\begin{array}{l}\text { A monitorização da farmacoterapia em recém- } \\
\text { nascidos de extremo baixo peso em unidade de } \\
\text { terapia intensiva neonatal de Fortaleza, Ceará, e } \\
\text { avaliação por meio de indicadores de processo } \\
\text { e resultado }\end{array}$ & Revisão Descritiva/Exploratório. & $\begin{array}{l}\text { O presente estudo mostrou ser possível a realização da monitorização da } \\
\text { farmacoterapia de recém-nascidos de extremo baixo peso, evidenciando o } \\
\text { papel do farmacêutico clínico na resolução e prevenção de problemas } \\
\text { relacionados aos medicamentos, contribuindo para a obtenção de uma } \\
\text { farmacoterapia segura e eficaz. }\end{array}$ \\
\hline Carvalho (2016). & $\begin{array}{l}\text { Proteger a saúde das crianças e garantir que } \\
\text { esses medicamentos sejam usados de forma } \\
\text { mais ética. }\end{array}$ & Estudo Descritivo Transversal. & $\begin{array}{l}\text { De imediato, para induzir o emprego ético de medicamentos off-Label, é } \\
\text { necessário que seu uso excepcional seja justificado clinicamente, ainda que } \\
\text { seja acompanhado de esclarecimento e consentimento dos responsáveis.14 } \\
\text { Essa medida pode ser tomada pelas unidades de saúde. A Agência Nacional de } \\
\text { Vigilância Sanitária (Anvisa), a exemplo do órgão regulador da União } \\
\text { Europeia, deveria estabelecer critérios e normas que induzam estudos } \\
\text { comparativos e mostrem a eficácia e segurança do uso de medicamentos em } \\
\text { crianças. Quando promissoras, as terapias devem ser testadas em ensaios } \\
\text { clínicos controlados e as bulas reformuladas. }\end{array}$ \\
\hline Marques (2017). & $\begin{array}{l}\text { Avaliar o perfil de uso seguro de medicamentos } \\
\text { em crianças internadas no setor de pediatria de } \\
\text { um hospital público de Brasília. }\end{array}$ & Revisão Experimental. & $\begin{array}{l}\text { Os resultados obtidos suscitam a necessidade de ponderação pelos diferentes } \\
\text { profissionais de saúde sobre o uso seguro de medicamentos na população } \\
\text { pediátrica. Para tanto, os apontamentos desta pesquisa podem servir para } \\
\text { direcionar ações relacionadas à segurança dos pacientes internados no } \\
\text { hospital, o que inclui a completude dos registros nos prontuários como, por } \\
\text { exemplo, dados de peso corporal, essenciais para a eficácia e segurança } \\
\text { terapêutica. }\end{array}$ \\
\hline
\end{tabular}


Research, Society and Development, v. 10, n. 15, e208101522896, 2021

(CC BY 4.0) | ISSN 2525-3409 | DOI: http://dx.doi.org/10.33448/rsd-v10i15.22896

\begin{tabular}{|c|c|c|c|}
\hline Paiva et al. (2017). & $\begin{array}{l}\text { Investigar uso off-Label de antimicrobianos na } \\
\text { pediatria. }\end{array}$ & Revisão Bibliográfica. & $\begin{array}{l}\text { O presente trabalho foi possível evidenciar o amplo uso off-label de } \\
\text { medicamentos antimicrobianos dentro da pediatria, uma vez que Revista de } \\
\text { Casos e Consultoria são escassos os ensaios clínicos de fármacos para essa } \\
\text { faixa etária sendo necessária a adaptação para a aplicabilidade dentro do } \\
\text { ambiente hospitalar. }\end{array}$ \\
\hline Manieiro et al. (2018). & $\begin{array}{l}\text { Analisar o perfil de utilização de medicamentos } \\
\text { em crianças de zero a cinco anos de idade. }\end{array}$ & Estudo Transversal. & $\begin{array}{l}\text { As crianças estudadas apresentam uma frequência elevada de uso de } \\
\text { medicamentos, devendo ser incentivadas ações que visem ao uso seguro e } \\
\text { racional de fármacos nessa população. }\end{array}$ \\
\hline Medeiros (2018). & $\begin{array}{l}\text { Realizar uma revisão da literatura sobre a } \\
\text { farmacologia pediátrica, evidenciando a } \\
\text { importância da atuação do farmacêutico clínico } \\
\text { para obtenção do êxito na farmacoterapia } \\
\text { infantil. }\end{array}$ & Revisão Bibliográfica. & $\begin{array}{l}\text { O farmacêutico clínico tem um papel crucial na farmacoterapia pediátrica, } \\
\text { sendo indispensável a sua participação como parte integrante na equipe de } \\
\text { saúde, realizando o acompanhamento farmacoterapêutico no intuito de reduzir } \\
\text { os efeitos indesejáveis, na escolha da forma farmacêutica mais adequada e no } \\
\text { ajuste posológico, melhorando a farmacoterapia, garantindo a segurança e a } \\
\text { qualidade de vida do paciente. }\end{array}$ \\
\hline Silva (2021). & $\begin{array}{l}\text { Analisar o papel do farmacêutico diante do } \\
\text { consumo de medicamentos off-Label. }\end{array}$ & Revisão Bibliográfica. & $\begin{array}{l}\text { Existem riscos potencializados no uso de medicamentos off-Label, devido à } \\
\text { falta de informações conclusivas sobre os agentes e fármacos inseridos no } \\
\text { medicamento. O farmacêutico pode ponderar riscos e benefícios quando se } \\
\text { opta pela prescrição off label. }\end{array}$ \\
\hline Diel et al. (2020). & $\begin{array}{l}\text { Estimar a prevalência de uso off-Label de } \\
\text { medicamentos segundo a idade em crianças de } \\
0 \text { a } 12 \text { anos no Brasil. }\end{array}$ & Estudo Transversal. & $\begin{array}{l}\text { O uso off-Label de medicamentos segundo a idade é comum na população } \\
\text { pediátrica brasileira, especialmente nas crianças menores de } 2 \text { anos de idade. } \\
\text { Políticas públicas de saúde que estimulem o desenvolvimento de pesquisas } \\
\text { sobre segurança e eficácia dos medicamentos disponíveis e usados como off- } \\
\text { label são urgentes e necessárias nesse grupo etário, bem como programas de } \\
\text { educação permanente voltadas aos prescritores e ações regulatórias, de } \\
\text { vigilância sanitária e de farmacovigilância. }\end{array}$ \\
\hline Da Gama (2021). & $\begin{array}{l}\text { Apresentar o desenvolvimento, construção e } \\
\text { validação de uma cartilha digital sobre estresse } \\
\text { e ansiedade voltada para acadêmicos do Ensino } \\
\text { Superior. }\end{array}$ & Estudo Transversal. & $\begin{array}{l}\text { Esse estudo reforça a necessidade do cuidado com a saúde mental dos } \\
\text { estudantes assim como ressalta a importância do desenvolvimento de } \\
\text { estratégias como um produto educacional para redução de fatores que possam } \\
\text { vir a prejudicar ou impedir a formação profissional dos indivíduos. }\end{array}$ \\
\hline
\end{tabular}

Fonte: Autoras, 2021. 


\section{Discussão}

Para Afonso (2015) o farmacêutico clínico, pelos seus conhecimentos sobre medicamentos e o seu papel no processo de distribuição de fármacos, encontra-se numa posição fundamental para a prevenção dos erros de medicação e contribuir para o uso correto da medicação. Entre as principais intervenções do farmacêutico clínico, incluem-se a prestação de informação acerca do medicamento aos restantes profissionais de saúde, quer sejam questões acerca do manuseamento ou de interações, sugestões acerca do tratamento e prevenção de erros de medicação.

Quatro fármacos de ação antimicrobiana figuraram entre os medicamentos mais prescritos entre os neonatos, o que está associado à ocorrência de infecção neonatal. Loureiro (2015) observou em seu estudo altas taxas de prescrição de antimicrobianos. O referido autor também constatou predominância de surfactante entre os itens mais prescritos na população estudada. As infecções hospitalares são mais frequentes e, geralmente, mais graves em recém-nascidos do que em crianças maiores e em adultos.

No que diz respeito às formulações pediátricas, os principais fatores que desestimulam a indústria farmacêutica a desenvolver medicamentos para crianças, contemplam: o número pequeno de sujeitos disponíveis para participar destas pesquisas; os elevados custos marginais; o mercado reduzido; e a complexidade ética desses estudos (Carvalho, 2016).

O estudo analisado por Marques (2017) em um hospital as crianças internadas com patologias desde hérnia umbilical a afecções mal curadas, administrando nas mesmas medicamentos durante esse período como: Ibuprofeno, cetoprofeno, paracetamol e dipirona, revelando a dipirona que é um analgésico e antitérmico com frequente uso no Brasil, foi o fármaco mais prescrito de forma off-Label nesta pesquisa tendo (12,7\%) de utilização por ser um medicamento de ação rápida é usado como primeira escolha na administração endovenosa, levando em consideração a melhora rápida do paciente.

Segundo o autor Paiva et al. (2017) a ampicilina sódica um dos antimicrobianos mais utilizados em âmbito hospitalar normalmente é administrada doses diferentes das preconizadas onde se diz ( 25 a $50 \mathrm{mg} / \mathrm{kg} / \mathrm{dia}$ ), em doses iguais, a cada seis a oito horas sendo prescritos um total de $150 \mathrm{mg} / \mathrm{kg}$ e considerando a necessidade do paciente, sua idade e características como peso. Diante disso, vale ressaltar que as formulações pediátricas necessitam de cálculos complexos para resultar em dosagem adequada. Por isso, faz-se essencial a presença do profissional farmacêutico em ambiente hospitalar para que haja a realização de ensaios em tempo hábil para atender a urgência da terapia clínica em Unidades de Terapia Intensiva.

Manieiro et al. (2018) verificaram de acordo com os vários estudos publicados, que são os recém-nascidos e lactentes quem recebem a maior fração de fármacos não aprovados, em regime offLabel e soluções extemporâneas, principalmente a nível hospitalar. Levando em consideração que os recém-nascidos e lactentes são particularmente vulneráveis as dificuldades práticas que se encontram quando se pretende administrar a dose de fármaco e a sua incapacidade de reportar quaisquer reações adversas, é necessário e urgente assegurar a eficácia e segurança de muitos fármacos atualmente prescritos nestes regimes para estes grupos etários.

Apesar de existirem riscos da prescrição off-Label para a população pediátrica em relação aos possíveis efeitos adversos, interação medicamentosa e intoxicação, a prática não é contraindicada, logo, é preciso estar atento sobre a incidência de reações adversas medicamentosas (Medeiros, 2018).

Na visão de Silva (2021), o farmacêutico é o profissional de saúde responsável por verificar a eficácia ou o fracasso terapêutico dos medicamentos prescritos. Isso pode acontecer por meio da orientação na hora da dispensação, como intuito de garantir a forma adequada de administração, assim como na consulta farmacêutica, em que é feito o acompanhamento do tratamento do paciente. Para que a orientação e o acompanhamento farmacêutico aconteçam com excelência, é preciso haver 
uma boa capacitação dos farmacêuticos abrangendo o tema, para que esses tenham um conhecimento adequado, sabendo reconhecer os medicamentos off-Label e equilibrar os riscos e benefícios.

Na pesquisa de Diel et al. (2020), a loratadina foi o terceiro medicamento mais prescrito e teve uma frequência de prescrição off-Label de $85,3 \%, 53,1 \%$ por frequência de administração, 25,0\% para idade inferior à recomendada e $21,9 \%$ por sobredose. O salbutamol, cuja frequência de prescrição foi 7,3\% (53), foi prescrito off-Label em 100,0\% das prescrições em uma indicação para faixa etária inferior à recomendada em 50,9\% e para uso em doses acima do recomendado pela bula em $49,1 \%$ casos.

No grupo dos pré-escolares, Da Gama (2021) destaca as prescrições de salbutamol, cuja função é um broncodilatador que facilita a melhora em pacientes com asma ou doenças nas vias aéreas possui um efeito benéfico e um adverso que é a presença de arritmias cardíacas, sendo necessária a observação do paciente mais efetiva durante a utilização e amoxicilina em doses acima das recomendadas, por ser um antimicrobiano o uso em demasia pode provocar uma resistência ao medicamento o tornando ineficaz pelos pacientes, devendo ser acima de tudo orientada a utilização nos pacientes.

\section{Considerações Finais}

Neste estudo, foi possível perceber que o uso de medicamentos off-Label ainda é uma prática bastante comum entre os prescritores e a posologia ainda continua sendo um tabu para quem administra esses medicamentos para crianças.

Nos consultórios farmacêuticos existem uma grande procura de responsáveis por crianças com algum tipo de enfermidade solicitando a prescrição de medicamentos cuja posologia está indicada para crianças maiores causando muitas vezes reações adversas.

O farmacêutico é essencial na farmacoterapia à pacientes pediátricos com uso de medicamentos off-Label, pois os mesmos requerem atenção à ocorrência de PRM's, má adesão à farmacoterapia utilizada e o médico prescritor deverá ser notificado para a perfeita recuperação do paciente.

Os pais e/ou responsáveis deverão ter orientações a todo instante dos perigos de administrar medicamentos para suas crianças, mesmo tentando ajudar pode agravar o estado clínico do paciente, pois a posologia pode não ser a indicada para as características fisiológicas da criança.

O uso desses medicamentos, quando necessário, deverá ser acompanhado de perto por um profissional que consiga reverter algum quadro de intoxicação, reações inesperadas, entre outras que o uso indiscriminado pode gerar.

Sendo assim, é importante que se tenha uma abordagem cada vez mais centrada no doente e não no fármaco. Nessa fase, a contribuição do farmacêutico é de suma importância para o paciente, onde o farmacêutico pode ponderar benefícios e riscos quando se opta pela prescrição off-Label.

\section{Implicações práticas}

Recomenda-se a necessidade de estudos complementares quanto a importância do farmacêutico na consulta à pacientes pediátricos com uso de medicamentos off-Label, a fim de enfatizar a importância da assistência na melhoria na qualidade do atendimento às crianças.

\section{Declaração de conflito de interesses}

Os autores declaram que a pesquisa foi conduzida na ausência de quaisquer relações comerciais ou financeiras que possam ser interpretados como um potencial conflito de interesse. 
Research, Society and Development, v. 10, n. 15, e208101522896, 2021

(CC BY 4.0) | ISSN 2525-3409 | DOI: http://dx.doi.org/10.33448/rsd-v10i15.22896

\section{Agradecimentos}

Agradeço primeiramente a Deus, a minha família que é minha base e a minha orientadora Daiane Pereira dos Santos Menese pelo apoio.

\section{Referências}

Afonso, A. M. M. (2015). Farmácia clínica em pediatria. Dissertação (Mestrado em Ciências Farmacêuticas) Universidade do Algarve, Faculdade de Ciências e Tecnologia Departamento de Química e Farmácia.

Camerini, F. G., Fassarella, C. S., de Mendonça Henrique, D., Assad, L. G., \& Radighieri, A. R. (2019). Gravidade das incompatibilidades medicamentosas em pacientes críticos: Uma revisão integrativa. Saúde Coletiva (Barueri), 9(51), 1907-1912.

Carvalho, M. L. (2016). O desafio do uso off label de medicamentos. Revista Paulista Pediatria. https://doi.org/10.1016/j.rppede.2015.12.007.

Correia, G. B. (2019). Elaboração de guia de diluição e estabilidade de medicamentos injetáveis vigentes em hospital materno infantil de Caicó-RN. Monografia (Especialização) - Curso de Residência Multiprofissional em Saúde Materno-infantil, Universidade Federal Rio Grande Norte.

da Gama, H. F. L. N. (2021). Saúde e aplicações interdisciplinares. v. 3.

de Paiva, E. C da Silva Miranda, C. C., da Silva, M. S., Alves, M. H. P., Silva, J. F. T., de Sousa Santos, A. B. A., ... \& Pierote, J. C. L. (2017). O uso off-label de antimicrobianos na pediatria. Revista de Casos e Consultoria, 12(1), e25203-e25203.

de Sousa, L. M. M., Marques-Vieira, C. M. A., Severino, S. S. P., \& Antunes, A. V. (2017). A metodologia de revisão integrativa da literatura em enfermagem. $N^{\circ} 21$ Série 2-Novembro 2017, 17.

Diel, J. D. A. C., Heineck, I., Santos, D. B. D., \& Pizzol, T. D. S. D. (2020). Uso off-label de medicamentos segundo a idade em crianças brasileiras: um estudo populacional. Revista Brasileira de Epidemiologia, 23. https://doi.org/10.1590/1980-549720200030.

dos Anjos Medeiros, I. A., \& Oliveira, F. S. farmacoterapia pediátrica: as particularidades da utilização de fármacos em pediatria.

https://doi.org/10.35572/rsc.v9i3.468.

Gonçalves, M. G., \& Heineck, I. (2016). Frequência de prescrições de medicamentos off label e não licenciados para pediatria na atenção primária à saúde em município do sul do Brasil. Revista Paulista de Pediatria, 34, 11-17.

Koszma, EIA, Bispo, AJB, Santana, IADO, \& Santos, CNODBD (2021). Uso de medicamentos off-label em unidade de terapia intensiva neonatal. Revista Paulista de Pediatria, 39. https://doi.org/10.1590/1984-0462/2021/39/2020063.

Loureiro, CV (2015). Monitorização da farmacoterapia em recém-nascidos de baixo peso extremo em unidade de terapia intensiva neonatal. Dissertação (Mestrado em Ciências Farmacêuticas) - Faculdade de Farmácia, Odontologia e Enfermagem, Universidade Federal do Ceará, Fortaleza.

Lubrano, R., Paoli, S., Bonci, M. et al. Acetaminophen administration in pediatric age: an observational prospective cross-sectional study. Ital J Pediatr 42, 20 (2016). https://doi.org/10.1186/s13052-016-0219-x

Lucena, P. S. D. (2018). Estudos sobre as compatibilidades de medicamentos administrados em Y em hospitais pediátricos. Trabalho de Conclusão de Curso em Farmácia, UFRGS.

Maniero, H. K., Martins, A. A., Melo, A. C., Paz, L. P. D. S., Schraiber, R. D. B., \& Galato, D. (2018). Uso de medicamentos em crianças de zero a cinco anos de idade residentes no município de Tubarão, Santa Catarina. Revista Paulista de Pediatria, 36, 437-44.

http://dx.doi.org/10.1590/1984-0462/;2018;36;4;00008.

Marques, L. R. (2017). Avaliação do uso seguro de medicamentos em pacientes pediátricos de um hospital público de Brasília/DF. Trabalho de Conclusão de Curso (Bacharelado em Farmácia)—Universidade de Brasília.

MEDEIROS, I. A. D. A. (2018). Farmacologia pediátrica: uma revisão sobre a importância do farmacêutico clínico na farmacoterapia infantil. Trabalho de Conclusão de Curso em Farmácia na UFCG. http://dspace.sti.ufcg.edu.br:8080/jspui/handle/riufcg/6664.

Nascimento, E. B. (2020). Serviço de atenção farmacêutica (SAF) em farmácia escola: uma revisão de literatura.

Martins, G., Molina, N., \& Uzam, C. D. P. P. (2017). Impacto dos medicamentos nas intoxicações em crianças. Revista da Universidade Ibirapuera. 
Research, Society and Development, v. 10, n. 15, e208101522896, 2021

(CC BY 4.0) | ISSN 2525-3409 | DOI: http://dx.doi.org/10.33448/rsd-v10i15.22896

Porto, D. T. G. (2015). Implantação de práticas seguras para distribuição e dispensação de medicamentos perigosos perigosos em um hospital oncológico da rede sentinela. Repositório Institucional da UFPB.

Silva, M. E. H., \& de Carvalho Abreu, C. R. (2021). Medicamentos off label. Revista JRG de Estudos Acadêmicos, 4(8), 300-308. https://doi.org/10.5281/zenodo.464112.

Silva Júnior, J. A. D. (2019). Atenção farmacêutica no uso racional de medicamentos como estratégia na promoção da saúde aos grupos pediátricos e geriátricos: Uma revisão integrativa. Trabalho de Conclusão de Curso (Graduação em Farmácia) - Universidade Federal do Amazonas, Itacoatiara.

Soares, F. R., \& Dadalto, L. (2020). Responsabilidade médica e prescrição off-label de medicamentos no tratamento da COVID-19. Revista IBERC, 3(2), 1-22. https://doi.org/10.37963/iberc.v3i2.112

Vieira, V. C., Costa, R. S., Lima, R. C. G., Queiroz, D. B., \& Medeiros, D. S. D. (2021). Prescrição de medicamentos off-label e sem licença para prematuros de unidade de terapia intensiva neonatal. Revista Brasileira de Terapia Intensiva, 33, 266-275. https://doi.org/10.5935/0103-507X.20210034. 